\title{
Níveis críticos e faixas de suficiência nutricional em laranjeira-pêra na Amazônia Central obtidas pelo método DRIS
}

\author{
Jairo Rafael Machado DIAS ${ }^{1}$, Carlos Alberto Franco TUCCI², Paulo Guilherme Salvador WADT², \\ Aldilane Mendonça da SILVA ${ }^{4}$, José Zilton Lopes SANTOS 5
}

\begin{abstract}
RESUMO
A avaliação do estado nutricional da laranjeira depende da definição de valores de referência que sejam adequados para refletir suas condiçóes nutricionais. Neste trabalho, objetivou-se determinar os valores de referências e avaliar o estado nutricional de laranjeiras-pêra em diversas glebas na Amazônia Central (municípios de Iranduba, Manacapuru, Manaus, Presidente Figueiredo e Rio Preto da Eva). Utilizou-se o Sistema Integrado de Diagnose e Recomendação de relaçóes multivariadas (DRIS) para estabelecer os valores de referência nutricional. O diagnóstico nutricional de 120 glebas comerciais de laranjeiras-pêra, enxertadas em limoeiro cravo foram avaliadas pelas faixas de suficiência definidas a partir do conjunto de plantas nutricionalmente equilibradas. Para os macronutrientes, as faixas de suficiência nutricional foram $\left(\mathrm{g} \mathrm{kg}^{-1}\right)$ : 28-30 (para nitrogênio, N); 1,6-1,7 (fósforo, P); 7-9 (potássio, K); 26-29 (cálcio, Ca); 3,4-4 (magnésio, Mg); 1,7-2 (enxofre, S) e para os micronutrientes (mg kg 1): 47-56 (boro, B); 8-10 (cobre, Cu); 84-93 (ferro, Fe); 12-13 (manganês, Mn); 14-16 (zinco, Zn). Para os macronutrientes, os níveis críticos foram $\left(\mathrm{g} \mathrm{kg}^{-1}\right): 28$ (para N); 1,6 (P); $7(\mathrm{~K}) ; 26(\mathrm{Ca}) ; 3,6(\mathrm{Mg}) ; 1,7(\mathrm{~S})$ e para os micronutrientes $\left(\mathrm{mg} \mathrm{kg}^{-1}\right)$ : $47(\mathrm{~B}) ; 8(\mathrm{Cu}) ; 84(\mathrm{Fe}) ; 12(\mathrm{Mn}) ; 14$ para Zn. Padrōes nutricionais obtidos pelo DRIS discordam das faixas de suficiência propostas pela literatura para maioria dos nutrientes. Em quase 50\% das glebas monitoradas, $\mathrm{P}, \mathrm{K}, \mathrm{Ca}, \mathrm{S}, \mathrm{B}, \mathrm{Cu}$ e Fe estão abaixo dos níveis críticos propostos neste trabalho. Isto sugere que os produtores de laranja na Amazônia Central deveriam atentar-se para estes elementos no planejamento das fertilizaçóes.
\end{abstract}

PALAVRAS-CHAVE: Citrus sinensis, estado nutricional, diagnose foliar, monitoramento nutricional.

\section{Critical levels and nutrient sufficiency ranges in orange of the Central Amazon determined by DRIS method}

\begin{abstract}
The nutritional status of orange trees depends of reference values that are appropriate to reflect their nutritional condition. The objective this work was to assess the nutrient reference values and evaluated the nutritional status of sweet orange trees in several orchard fields of Central Amazonia (municipalities of Iranduba, Manacapuru, Manaus, Presidente Figueiredo and Rio Preto da Eva). We used the Diagnosis and Recommendation Integrated System for multivariate relation (DRIS) method to establish the nutrient reference values. The nutritional status of 120 commercial orchards of orange grafted on rangpur lime was evaluated by the sufficiency ranges defined from the set of nutritionally balanced plants. For macronutrients, the nutritional sufficiency ranges were $\left(\mathrm{g} \mathrm{kg}^{-1}\right)$ : 28-30 (for nitogen, N); 1.6-1.7 (phosphorus, P); 7-9 (potassium, K); 26-29 (calcium, Ca); 3.6-4.0 (magnesium, $\mathrm{Mg}$ ); 1.7-2.0 (sulfur - S) and for micronutrients $\left(\mathrm{mg} \mathrm{kg}^{-1}\right)$ : $47-56$ (boron, B); 8-10 (copper, Cu); 84-93 (iron, Fe); 12-13 (manganese, Mn); 14-16 (zinc, Zn). For macronutrients, the critical levels were ( $\left.\mathrm{g} \mathrm{kg}^{-1}\right): 28$ (for N); 1.6 (P); 7 (K); 26 (Ca); 3.6 $(\mathrm{Mg}) ; 1.7(\mathrm{~S})$ and for micronutrients $\left(\mathrm{mg} \mathrm{kg}^{-1}\right): 47(\mathrm{~B}) ; 8(\mathrm{Cu}) ; 84(\mathrm{Fe}) ; 12(\mathrm{Mn})$ and 14 for $\mathrm{Zn}$. Nutritional standards obtained by the DRIS methods are in disagreement with the normal ranges proposed in the literature for most nutrients. In about $50 \%$ of orchards, the elements P, K, Ca, S, B, Cu e Fe were found below the critical levels. This suggests that orange producers in Central Amazonia should pay more attention to these elements when planning fertilization practices.
\end{abstract}

KEYWORDS: Citrus sinensis, nutritional status, foliar analysis, nutritional monitoring.

\footnotetext{
1 Programa de Pós-Graduação em Agronomia Tropical (PPG-AT), Universidade Federal do Amazonas, UFAM - Av. General Rodrigo Octávio Jordão Ramos, 3000, Campus Universitário, Coroado I, Setor Sul, Bloco A, Coordenação do PPG-AT, CEP: 69.077-000, Manaus - AM, Brasil. E-mail: jairorafaelmdias@hotmail.com

2 PPG-AT/UFAM. E-mail: ctucci@ufam.edu.br

${ }^{3}$ Empresa Brasileira de Pesquisa Agropecuária, Centro de Pesquisa Agroflorestal do Acre - Rodovia BR 364, Caixa Postal 321, km 14, CEP: 69.900-056, Rio Branco - AC, Brasil. E-mail: paulogswadt@dris.com.br

${ }^{4}$ PPG-AT/UFAM. E-mail: aldile001@hotmail.com

${ }^{5}$ PPG-AT/UFAM. E-mail: ziltton@yahoo.com.br
} 


\section{INTRODUÇÃO}

O estado nutricional de uma planta pode ser estabelecido comparando a concentraçáo de determinado nutriente no tecido de um determinado órgáo, como folhas, seiva ou pecíolos, com a concentraçáo do mesmo nutriente em plantas sadias e produtivas, ao que denomina-se valor de referência ou valor nutricional padrão. Quando o valor padrão corresponde ao teor do nutriente a partir do qual o nível produtivo da cultura será igual ou maior que $90 \%$ da produtividade máxima, denomina-se de nível crítico (NC). Comumente se adotada uma amplitude de valores com nível produtivo igual ou superior a $90 \%$ da produtividade máxima, denomina-se por faixas de suficiência (FS) (Kurihara et al. 2005).

O diagnóstico nutricional comparando-se os teores dos nutrientes com um dado NC ou FS não considera as interaçôes entre os nutrientes ou as condiçôes de crescimento das plantas, motivo pelo qual faz-se necessário que todas as demais condiçôes, exceto o nutriente em análise, sejam controlados e mantidos em condiçôes de disponibilidade ótima. Por isto, as condições de crescimento das plantas a serem avaliadas devem ser semelhantes àquelas utilizadas para a obtenção da curva de calibração, utilizadas para a obtenção dos valores padrōes (NC ou FS), no que diz respeito às condiçóes edafoclimáticas, à idade das plantas e do tecido, ao tipo de material genético, à posiçáo do tecido na planta e disponibilidade dos demais nutrientes (Fageria et al. 2009).

No Brasil, os valores de referência nutricional para laranjeiras limitam-se às condiçôes ecofisiológicas predominantes no estado de São Paulo (Malavolta et al. 1994; Quaggio et al. 2005), apesar de muitas vezes serem extrapoladas para outros Estados (Santana et al. 2007; Fernandes et al. 2010). Estes valores de referência não regionalizados podem resultar em imprecisóes na avaliação do estado nutricional das plantas cultivadas (Lana et al. 2010).

Devido à complexidade para a obtenção dos valores padrôes para a avaliação do estado nutricional em culturas comerciais, uma alternativa tem sido a derivaçáo dos valores padróes a partir de plantas consideradas nutricionalmente equilibradas pelo método do DRIS, como já obtido para outras culturas, incluindo laranjeiras (Santana et al. 2008; Camacho et al. 2012).

$\mathrm{Na}$ Amazônia central, a citricultura consiste de uma importante atividade para a economia regional, com índices elevados de crescimento nos últimos dez anos, superando 2,8 mil hectares plantados. Neste período, o Amazonas assumiu posiçáo de destaque, como segundo maior produtor da regiáo Norte (IBGE 2011). Contudo mesmo a laranjeira-pêra sendo bem adaptada às condiçóes edafoclimáticas locais, informaçōes sobre seu estado nutricional e demanda de nutrientes ainda são carentes na regiấo, fato que contribui para o baixo nível tecnológico desta atividade. Neste sentido, objetivou-se determinar padróes nutricionais e avaliar o estado nutricional de laranjeiras-pêra no estado do Amazonas, utilizando o método DRIS.

\section{MATERIAL E MÉTODOS}

Cento e vinte glebas comerciais de laranjeiras-pêra (Citrus sinensis $\mathrm{L}$. Osbeck) enxertadas em limoeiro cravo (Citrus limonia Osbeck), com população de 208 a 408 plantas ha ${ }^{-1} \mathrm{e}$ idade entre 8 a 15 anos, amostradas na região produtora de citros no Amazonas, nos municípios: Iranduba ( $03^{\circ} 17^{\prime} 06^{\prime \prime}$ $\mathrm{S}$ e $\left.60^{\circ} 11^{\prime} 09^{\prime \prime} \mathrm{W}\right)$, Manacapuru (03 $17^{\circ} 59^{\prime \prime} \mathrm{S}$ e $60^{\circ} 37^{\prime}$ $\left.14^{\prime \prime} \mathrm{W}\right)$; Manaus $\left(03^{\circ} 06^{\prime} 00^{\prime \prime} \mathrm{S}\right.$ e $\left.60^{\circ} 01^{\prime} 00^{\prime \prime} \mathrm{W}\right)$, Presidente Figueiredo ( $02^{\circ} 01^{\prime} 02^{\prime \prime} \mathrm{S}$ e $\left.60^{\circ} 01^{\prime} 30^{\prime \prime} \mathrm{W}\right)$ e Rio Preto da Eva $\left(02^{\circ} 41^{\prime} 56^{\prime \prime} \mathrm{S}\right.$ e $\left.59^{\circ} 42^{\prime} 00^{\prime \prime} \mathrm{W}\right)$ foram monitoradas quanto ao estado nutricional, entre os meses de fevereiro de 2010 a abril de 2011. As características do solo nas áreas estudadas estão contidas da Tabela 1.

O clima da regiáo classifica-se como Tropical Chuvoso - Af (Köppen), com temperatura média anual de $26^{\circ} \mathrm{C}$ e precipitação pluvial média de $2.550 \mathrm{~mm}$ ano $^{-1}$. O período chuvoso compreende os meses de dezembro a abril, com acúmulo de chuvas no primeiro trimestre do ano e o período mais quente compreende os meses de agosto e outubro (Sipam 2005).

Para cada gleba monitorada foram amostradas aleatoriamente vinte e cinco árvores, onde coletou-se um total de cem folhas recém amadurecidas, tomadas na terceira posição de lançamento a partir do ápice de ramos com fruto de seis meses de idade e diâmetro entre 2 e $4 \mathrm{~cm}$, na face das árvores referentes aos quatro pontos cardeais e sempre na altura mediana da planta. O material vegetal coletado foi acondicionado em sacos de papel e transportado para o laboratório, onde foram lavados, secados, moídos e submetidas às analises dos teores totais de $\mathrm{N}, \mathrm{P}, \mathrm{K}, \mathrm{Ca}, \mathrm{Mg}, \mathrm{S}, \mathrm{B}, \mathrm{Cu}, \mathrm{Fe}$, Mn e Zn (Malavolta et al. 1997).

Após obtenção dos dados analíticos das concentraçôes foliares, utilizou-se o método DRIS de relaçôes multivariadas para identificação das glebas nutricionalmente equilibradas (populaçáo de referência). Para tanto, os teores dos nutrientes foram ajustados para decagrama por quilograma $\left(\mathrm{dag} \mathrm{kg}^{-1}\right)$.

A seguir, calculou-se o valor do complemento dos nutrientes para o total da biomassa da folha, denominado de valor $\mathrm{R}$, conforme a expressão: $\mathrm{R}=100-(\mathrm{vN}+\mathrm{vP}+\mathrm{vK}+$ $\mathrm{vCa}+\mathrm{vMg}+\mathrm{vS}+\mathrm{vB}+\mathrm{vCu}+\mathrm{vFe}+\mathrm{vMn}+\mathrm{vZn})$. Onde, $\mathrm{R}$ representa o conteúdo da matéria e a massa correspondente aos demais nutrientes náo avaliados e, $\mathrm{vN}, \mathrm{vP}, \mathrm{vK}, \mathrm{vCa}, \mathrm{vMg}$, vS, vB, vCu, vFe, vMn e vZn são os teores de $\mathrm{N}, \mathrm{P}, \mathrm{K}, \mathrm{Ca}$, $\mathrm{Mg}, \mathrm{S}, \mathrm{B}, \mathrm{Cu}, \mathrm{Fe}, \mathrm{Mn}$ e $\mathrm{Zn}$, respectivamente, expressos em dag $\mathrm{kg}^{-1}$. Ainda, em cada amostra foliar calculou-se a média 
Tabela 1 - Valores médios, desvio padrão (DP), mínimo (Min.) e máximo (Máx.) das características do solo nas glebas de laranjeira-pêra, nos municípios: Iranduba, Manacapuru, Manaus, Presidente Figueiredo e Rio Preto da Eva, estado do Amazonas.

\begin{tabular}{|c|c|c|c|c|c|c|c|c|c|c|}
\hline \multirow[t]{2}{*}{ Valores } & \multirow[t]{2}{*}{$\mathrm{pH}$} & $\mathrm{H}+\mathrm{Al}$ & $\mathrm{Al}$ & $\mathrm{Ca}$ & $\mathrm{Mg}$ & $P$ & $\frac{\mathrm{K}}{\left(m m^{-3}\right.}$ & $S$ & \multirow[t]{2}{*}{$\begin{array}{c}\mathrm{MO} \\
\mathrm{g} \mathrm{kg}^{-1}\end{array}$} & \multirow[t]{2}{*}{$\begin{array}{c}\text { Arg. } \\
\%\end{array}$} \\
\hline & & \multicolumn{4}{|c|}{$\left(\mathrm{cmol}_{\mathrm{c}} \mathrm{dm}^{-3}\right)$} & \multicolumn{3}{|c|}{$\left(\mathrm{mg} \mathrm{dm}^{-3}\right)$} & & \\
\hline \multicolumn{11}{|c|}{ Iranduba } \\
\hline Média & 4,99 & 1,76 & 0,58 & 1,68 & 1,05 & 3,98 & 37,75 & 21,62 & 26,46 & 46 \\
\hline DP & 0,69 & 0,49 & 0,30 & 0,55 & 0,34 & 1,11 & 7,33 & 3,45 & 8,15 & 1,27 \\
\hline Mín. & 4,16 & 1,10 & 0,15 & 1,00 & 0,70 & 2,13 & 30,00 & 17,35 & 14,70 & 43 \\
\hline Máx. & 6,80 & 2,71 & 1,20 & 2,85 & 1,85 & 6,93 & 54,00 & 26,61 & 38,00 & 48 \\
\hline \multicolumn{11}{|c|}{ Manacapuru } \\
\hline Média & 4,59 & 3,73 & 1,58 & 1,12 & 0,77 & 5,15 & 26,88 & 27,22 & 22,73 & 52 \\
\hline DP & 0,43 & 0,19 & 0,34 & 0,14 & 0,17 & 0,96 & 4,30 & 3,19 & 4,42 & 2,28 \\
\hline Mín. & 4,02 & 3,45 & 0,60 & 0,90 & 0,55 & 3,49 & 22,00 & 20,29 & 14,70 & 48 \\
\hline Máx. & 4,99 & 3,99 & 2,05 & 1,30 & 1,15 & 6,46 & 38,00 & 31,45 & 29,40 & 55 \\
\hline \multicolumn{11}{|c|}{ Manaus } \\
\hline Média & 4,98 & 1,53 & 0,40 & 2,06 & 1,11 & 5,19 & 35,00 & 24,22 & 43,79 & 55 \\
\hline DP & 0,33 & 0,27 & 0,16 & 0,40 & 0,13 & 1,52 & 5,06 & 6,24 & 2,13 & 7,04 \\
\hline Mín. & 4,45 & 1,00 & 0,15 & 1,50 & 0,90 & 3,06 & 26,00 & 15,25 & 40,70 & 46 \\
\hline Máx. & 5,65 & 1,91 & 0,75 & 2,80 & 1,40 & 7,96 & 48,00 & 36,14 & 48,70 & 65 \\
\hline \multicolumn{11}{|c|}{ Presidente Figueiredo } \\
\hline Média & 4,60 & 2,13 & 0,68 & 1,56 & 0,88 & 3,21 & 31,38 & 28,39 & 20,54 & 47 \\
\hline DP & 0,30 & 0,21 & 0,21 & 0,31 & 0,14 & 0,50 & 3,77 & 6,03 & 7,51 & 5,81 \\
\hline Mín. & 4,13 & 1,75 & 0,25 & 1,15 & 0,60 & 2,33 & 22,00 & 17,78 & 13,50 & 37 \\
\hline Máx. & 4,98 & 2,60 & 0,95 & 2,05 & 1,10 & 3,93 & 38,00 & 38,09 & 36,40 & 54 \\
\hline \multicolumn{11}{|c|}{ Rio Preto da Eva } \\
\hline Média & 5,06 & 1,83 & 0,86 & 1,54 & 0,83 & 4,33 & 36,63 & 23,77 & 19,71 & 72 \\
\hline DP & 0,26 & 0,52 & 0,48 & 0,23 & 0,16 & 0,29 & 8,29 & 3,35 & 5,41 & 2,13 \\
\hline Mín. & 4,91 & 1,10 & 0,20 & 1,05 & 0,55 & 4,01 & 20,00 & 18,29 & 13,20 & 68 \\
\hline Máx. & 5,84 & 2,90 & 1,75 & 1,80 & 1,10 & 4,96 & 48,00 & 28,09 & 28,80 & 75 \\
\hline
\end{tabular}

geométrica (mGeo) dos teores nutricionais, pela expressão: $\mathrm{mGeo}=(\mathrm{vN} \times \mathrm{vP} \times \mathrm{vK} \times \mathrm{vCa} \times \mathrm{vMg} \times \mathrm{vS} \times \mathrm{vB} \times \mathrm{vCu} \times \mathrm{vFe}$ $\mathrm{x} v \mathrm{Mn} \mathrm{x} v \mathrm{Zn} \times \mathrm{R})^{(1 / 12)}$

Em seguinda, para cada nutriente, determinou-se sua respectiva variável multinutriente $(\mathrm{zX})$ pelo logarítimo neperiano do quociente entre vX e a média geométrica ( $\mathrm{mGeo}$ ) dos teores nutricionais, pela expressão: $\mathrm{zX}=\ln (\mathrm{vX} / \mathrm{mGeo})$, em que $\mathrm{zX}$ representa o valor da relação multivariada de cada um dos nutrientes avaliados (zN, zP, zK, zCa, zMg, zS, zB, zCu, zFe, zMn e zZn)

Com os valores das relaçóes multivariadas de cada gleba, calculou-se para o conjunto da população monitorada os parâmetros descritivos: média aritmética $(\mathrm{mX})$ e desvio padrão $(s \mathrm{X})$, determinando-se as normas DRIS de relaçóes multivariadas. A utilização de todo o conjunto de dados para a obtenção das normas DRIS tem como objetivo aumentar a representatividade das estimativas populacionais pelo aumento do tamanho da amostra (Beverly 1993).

Obtida as normas DRIS, os índices DRIS multivariados para cada nutriente na respectiva gleba, foi calculado pela relação multivariada log-centrada (Parent 2011): I_X $=(Z x-$ $\mathrm{mX} / \mathrm{sX}$. Em que: I_X, mX e sX representam o índice DRIS de relação multivariada, a norma média e a norma desvio padrão para os nutrientes N, P, K, Ca, Mg, S, B, Cu, Fe, Mn e Zn.

Calculou-se também o índice de balanço nutricional médio $(\mathrm{IBNm})$ pela expressão: $\mathrm{IBNm}=\left(\left|\mathrm{I} \_\mathrm{N}\right|+\left|\mathrm{I} \_\mathrm{P}\right|\right.$ $+\left|\mathrm{I} \_\mathrm{K}\right|+\left|\mathrm{I} \_\mathrm{Ca}\right|+\left|\mathrm{I} \_\mathrm{Mg}\right|+\left|\mathrm{I} \_\mathrm{S}\right|+\left|\mathrm{I} \_\mathrm{B}\right|+$ $\left.\left|\mathrm{I} \_\mathrm{Cu}\right|+\left|\mathrm{I} \_\mathrm{Fe}\right|+\left|\mathrm{I} \_\mathrm{Mn}\right|+\left|\mathrm{I} \_\mathrm{Zn}\right|\right) / 11$. O nutriente foi considerado nutricionalmente equilibrado quando seu índice DRIS, em módulo foi menor que o IBNm (Wadt 2005).

Para cada nutriente, a partir dos teores médios nutricionais da população de referência, respectivo ao elemento em questão, determinou-se o intervalo de confiança (IC), a partir da expressão: $\mathrm{IC}=\mathrm{mX} \pm$ t $\alpha . s_{\mathrm{mx}}$. Em que, $\mathrm{mX}$ e $s_{\mathrm{mX}}$ representam o teor médio e desvio padrão, respectivamente para os nutrientes $\mathrm{N}, \mathrm{P}, \mathrm{K}, \mathrm{Ca}, \mathrm{Mg}, \mathrm{S}, \mathrm{B}, \mathrm{Cu}, \mathrm{Fe}, \mathrm{Mn}$ e $\mathrm{Zn}$ nas glebas nutricionalmente equilibradas e, t $\alpha$ é o valor de $t$ bilateral, a $1 \%$, com $\mathrm{n}-1$, sendo $\mathrm{n}=$ número total de dados.

Considerou-se como FS a amplitude do IC expressando-se os resultados em gramas por quilograma $\left(\mathrm{g} \mathrm{kg}^{-1}\right)$ e miligramas por quilograma $\left(\mathrm{mg} \mathrm{kg}^{-1}\right)$ para os macro e micronutrientes, respectivamente. Valores abaixo e acima dos limites inferior e superior do IC representam teores nutricionais deficiente e alto (consumo de luxo e, ou, toxidez), respectivamente. 
Dado que NC representa o teor do nutriente a partir do qual a probabilidade de resposta ao aumento da sua disponibilidade não resulta em maior eficiência econômica e, que o teor médio representa o ótimo biológico para a disponibilidade do nutriente, adotou-se o limite inferior do IC de cada um dos nutrientes para obtenção do NC que por si, corresponde ao limite entre teores considerados deficientes e suficientes.

As frequências observadas pela distribuição percentual das 120 glebas em relaçáo ao estado nutricional (deficiente, normal e alto), a partir da avaliação por FS propostas neste trabalho foram contrastadas pela frequência esperada, diagnosticada por padrôes nutricionais para laranjeiras disponíveis na literatura (Malavolta et al. 1994; Quaggio et al. 2005) pelo teste quiquadrado $\left(\chi^{2}\right)$, ao nível de $1 \%$ de probabilidade.

\section{RESULTADOS}

As normas DRIS de relações multivariadas estão apresentadas na Tabela 2. Com base neste conjunto de normas, das 120 glebas de laranjeiras-pêra monitoradas, 72 , $68,46,59,60,71,66,77,83,70$ e 72 foram consideradas nutricionalmente equilibradas para $\mathrm{N}, \mathrm{P}, \mathrm{K}, \mathrm{Ca}, \mathrm{Mg}, \mathrm{S}, \mathrm{B}$, $\mathrm{Cu}, \mathrm{Fe}, \mathrm{Mn}$ e $\mathrm{Zn}$, respectivamente (Tabela 3).

As distribuiçóes das frequências para o número de glebas nas classes de insuficiência, equilíbrio e excesso nutricional foram semelhantes para maioria dos nutrientes, sendo a exceçấo $\mathrm{K}$, estando sob situaçấo de desequilíbrio nutricional na maioria das glebas estudadas (Tabela 3).

Nas glebas com plantas nutricionalmente equilibradas a amplitude dos teores nutricionais para os macronutrientes indicam serem $\mathrm{N}, \mathrm{Ke}$ Ca os elementos requeridos em maiores quantidades nos tecidos foliares. Já $\mathrm{P}, \mathrm{Mg}$ e $\mathrm{S}$ são exigidos praticamente na mesma ordem de grandeza (1,3 a $\left.5 \mathrm{~g} \mathrm{~kg}^{-1}\right)$ (Tabela 4).

A distribuição das frequências entre as classes nutricionais (deficiente, normal e alto), a partir dos resultados deste trabalho foram distintas para todos os nutrientes quando comparado aos valores críticos propostos pela literatura. Entretanto quando a distribuição das frequências nas classes nutricionais, pelas FS sugeridas pela literatura foram contrastadas entre si, a proporçáo de glebas deficientes, normais e com altos teores nutricionais foram semelhantes para N, K, S e Mn (Tabela 5).

Tabela 2 - Normas DRIS: média e desvio padrão das relações multivariadas log centradas para N, P, K, Ca, Mg, S, B, Cu, Fe, Mn e Zn para laranjeirapêra cultivada na Amazônia Central.

\begin{tabular}{llcccccccccc}
\hline Parâmetro & zN & zP & zK & zCa & zMg & zS & zB & zCu & zFe & zMn & zZn \\
\hline Média & 3,42 & 0,57 & 1,96 & 3,39 & 1,41 & 0,65 & $-2,98$ & $-4,54$ & $-2,37$ & $-4,32$ & $-4,08$ \\
Desvio & 0,15 & 0,23 & 0,49 & 0,26 & 0,37 & 0,21 & 0,35 & 0,66 & 0,47 & 0,33 & 0,23 \\
padrão & & &
\end{tabular}

Tabela 3 - Distribuição de frequência das 120 glebas de laranjeiras-pêra cultivadas na Amazônia central sob estado de insuficiência, equilíbrio e excesso nutricional a partir do DRIS de relações multivariadas para N, P, K, $\mathrm{Ca}, \mathrm{Mg}, \mathrm{S}, \mathrm{B}, \mathrm{Cu}, \mathrm{Fe}, \mathrm{Mn}$ e Zn, frequencia esperada de glebas em cada estado nutricional (FrEsp), valor calculado do teste de qui-quadrado $\left(\chi^{2}\right)$ e significância do teste qui-quadrado.

\begin{tabular}{cccccc}
\hline Nutriente & Insuficiência & Equilíbrio & Excesso & $\chi^{2}$ & Significância \\
\hline $\mathrm{N}$ & 16 & 60 & 24 & 1,2 & $56 \%$ \\
$\mathrm{P}$ & 23 & 57 & 20 & 0,6 & $74 \%$ \\
$\mathrm{~K}$ & 28 & 38 & 34 & 14,7 & $0 \%$ \\
\hline $\mathrm{Ca}$ & 26 & 49 & 25 & 0,7 & $71 \%$ \\
\hline $\mathrm{Mg}$ & 20 & 50 & 30 & 2,1 & $35 \%$ \\
\hline $\mathrm{S}$ & 22 & 59 & 19 & 0,9 & $64 \%$ \\
\hline $\mathrm{B}$ & 22 & 55 & 23 & 0,6 & $76 \%$ \\
\hline $\mathrm{Cu}$ & 18 & 64 & 18 & 3,5 & $18 \%$ \\
\hline $\mathrm{Fe}$ & 22 & 69 & 9 & 4,7 & $10 \%$ \\
\hline $\mathrm{Mn}$ & 20 & 58 & 22 & 0,6 & $74 \%$ \\
\hline $\mathrm{Zn}$ & 21 & 60 & 19 & 1,6 & $45 \%$ \\
\hline $\mathrm{FrEsp}$ & 21,6 & 56,3 & 22,1 & & \\
\hline
\end{tabular}

Todas as FS utilizadas para interpretação do estado nutricional foram unânimes em apontar alto teor foliar de $\mathrm{N}$ na maioria das glebas monitoradas. Já o $\mathrm{P}$ foi considerado deficiente em quase metade destas mesmas glebas, utilizandose das FS descritas neste trabalho. De forma generalizada, as FS para K propostas pela literatura foram capazes de detectar maior número de glebas em estado de deficiência nutricional, superando $74 \%$ nas glebas avaliadas por todos autores da literatura, indiscriminadamente, diferente das faixas estimadas neste trabalho que apontaram menos da metade das glebas sob estado de deficiência nutricional (Tabela 5).

Tabela 4 - Valores mínimo, máximo, desvio padrão (DP), nível crítico (NC) e faixas de suficiência (FS) dos teores foliares de N, P, K, Ca, Mg, S, B, Cu, $\mathrm{Fe}, \mathrm{Mn}$ e $\mathrm{Zn}$ em glebas de laranjeiras-pêra nutricionalmente equilibradas cultivadas na Amazônia Central pelo uso do DRIS de relações multivariadas ( $n=$ números de glebas envolvidas).

\begin{tabular}{lcccccc}
\hline Nutrientes & Mínimo & Máximo & $\mathrm{DP}$ & $\mathrm{n}$ & $\mathrm{NC}$ & $\mathrm{FS}$ \\
\hline $\mathrm{N}\left(\mathrm{g} \mathrm{kg}^{-1}\right)$ & 23 & 35 & 3 & 72 & 28 & $28-30$ \\
$\mathrm{P}\left(\mathrm{g} \mathrm{kg}^{-1}\right)$ & 1,3 & 2,0 & 0,2 & 68 & 1,6 & $1,6-1,7$ \\
$\mathrm{~K}\left(\mathrm{~g} \mathrm{~kg}^{-1}\right)$ & 3 & 13 & 2 & 46 & 7 & $7-9$ \\
$\mathrm{Ca}\left(\mathrm{g} \mathrm{kg}^{-1}\right)$ & 20 & 43 & 4 & 59 & 26 & $26-29$ \\
$\mathrm{Mg}\left(\mathrm{g} \mathrm{kg}^{-1}\right)$ & 2,1 & 5,0 & 0,7 & 60 & 3,6 & $3,6-4,0$ \\
$\mathrm{~S}\left(\mathrm{~g} \mathrm{~kg}^{-1}\right)$ & 1,3 & 2,8 & 0,3 & 71 & 1,7 & $1,7-2,0$ \\
$\mathrm{~B}\left(\mathrm{mg} \mathrm{kg}^{-1}\right)$ & 31 & 100 & 13 & 66 & 47 & $47-56$ \\
$\mathrm{Cu}\left(\mathrm{mg} \mathrm{kg}^{-1}\right)$ & 5 & 18 & 3 & 77 & 8 & $8-10$ \\
$\mathrm{Fe}\left(\mathrm{mg} \mathrm{kg}^{-1}\right)$ & 54 & 130 & 15 & 83 & 84 & $84-93$ \\
$\mathrm{Mn}\left(\mathrm{mg} \mathrm{kg}^{-1}\right)$ & 7 & 21 & 3 & 70 & 12 & $12-13$ \\
$\mathrm{Zn}\left(\mathrm{mg} \mathrm{kg}^{-1}\right)$ & 11 & 25 & 3 & 72 & 14 & $14-16$ \\
\hline
\end{tabular}


Tabela 5 - Frequência com que as 120 glebas de laranjeiras-pêra cultivadas da Amazônia Central apresentam estado deficiente, normal e alto teor nutricional pelo uso das faixas de suficiência por dois autores e por este trabalho.

\begin{tabular}{|c|c|c|c|c|c|c|c|c|c|c|c|}
\hline \multirow{2}{*}{$\begin{array}{l}\text { Estado } \\
\text { Nutricional }\end{array}$} & $\mathrm{N}$ & P & $\mathrm{K}$ & $\mathrm{Ca}$ & $\mathrm{Mg}$ & S & B & $\mathrm{Cu}$ & $\mathrm{Fe}$ & $\mathrm{Mn}$ & $\mathrm{Zn}$ \\
\hline & \multicolumn{11}{|c|}{ Frequencia } \\
\hline & \multicolumn{11}{|c|}{ Malavolta et al. $(1994)^{1}$} \\
\hline Deficiente & 3 & 3 & 75 & 81 & 9 & 67 & 72 & 65 & 93 & 97 & 90 \\
\hline Normal & 12 & 59 & 22 & 12 & 9 & 32 & 27 & 24 & 5 & 2 & 9 \\
\hline Alto & 85 & 37 & 3 & 7 & 82 & 1 & 1 & 11 & 2 & 1 & 1 \\
\hline \multirow[t]{2}{*}{$\chi^{2 \text { (1 vs. 2) }}$} & $0,1^{\text {ns }}$ & $0^{\star \star}$ & $0,1^{\text {ns }}$ & $0^{* *}$ & $0^{* *}$ & $1^{\mathrm{ns}}$ & $0^{* *}$ & $0^{\star *}$ & $0^{\star *}$ & $0,6^{\text {ns }}$ & $0^{* *}$ \\
\hline & \multicolumn{11}{|c|}{ Quaggio et al. (2005)² } \\
\hline Deficiente & 2 & 3 & 75 & 80 & 19 & 67 & 49 & 5 & 3 & 98 & 97 \\
\hline Normal & 20 & 39 & 24 & 17 & 30 & 32 & 50 & 60 & 87 & 1 & 2 \\
\hline Alto & 78 & 58 & 1 & 3 & 51 & 1 & 1 & 35 & 10 & 1 & 1 \\
\hline \multirow[t]{2}{*}{$\chi^{2 \text { (2vs.3) }}$} & $0^{* *}$ & $0^{* *}$ & $0^{* *}$ & $0^{* *}$ & $0^{* *}$ & $0^{* *}$ & $0^{* *}$ & $0^{* *}$ & $0^{* *}$ & $0^{* *}$ & $0^{* *}$ \\
\hline & \multicolumn{11}{|c|}{ Este trabalho ${ }^{3}$} \\
\hline Deficiente & 22 & 42 & 41 & 39 & 36 & 47 & 47 & 48 & 51 & 39 & 39 \\
\hline Normal & 28 & 12 & 16 & 22 & 13 & 17 & 15 & 17 & 13 & 18 & 22 \\
\hline Alto & 50 & 46 & 43 & 39 & 51 & 36 & 37 & 35 & 36 & 43 & 39 \\
\hline$\chi^{2 \text { (1 vs. 3) }}$ & $0^{* *}$ & $0^{* *}$ & $0^{* *}$ & $0^{* *}$ & $0^{* *}$ & $0^{* *}$ & $0^{* *}$ & $0^{* *}$ & $0^{* *}$ & $0^{* *}$ & 0 ** \\
\hline
\end{tabular}

$\chi^{2}=$ Cálculo do qui-quadrado; " ns $\mathrm{e}^{* *}=$ não significativo e significativo a $1 \%$ pelo teste $\mathrm{F}$.

De forma semelhante ao $\mathrm{K}$, o Ca foi o elemento apontado em mais de $79 \%$ das glebas sob deficiência a partir de FS definidas pela literatura. Já o $\mathrm{Mg}$ independente do padrão nutricional utilizado para interpretação do teor foliar foi apontado sob excesso na maioria das glebas monitoradas. Para $S$ tanto os padróes nutricionais sugeridos pela literatura quanto as faixas estimadas neste trabalho indicaram ser este $\mathrm{o}$ elemento mais comumente encontrado na classe de deficiência nutricional (Tabela 5).

A FS proposta neste trabalho apontaram $\mathrm{Fe}$ e $\mathrm{Cu}$ como nutrientes frequentemente sob estado de deficiência nutricional $(51 \%$ e $48 \%$, respectivamente das glebas monitoradas). Entretanto quanto se utiliza o método DRIS para avaliaçáo do estado nutricional, observa-se que estes mesmos elementos foram considerados frequentemente equilibrados (Tabela 3).

Para $\mathrm{Mn}$ e $\mathrm{Zn}$ as FS definidas pela literatura apontam deficiência generalizada, estando acima de $96 \%$ e $89 \%$, respectivamente nas glebas avaliadas. Já a quantidade de glebas deficientes em $\mathrm{B}$ definidas pela FS descritas neste trabalho foi semelhante aos resultados proporcionado pela faixa crítica proposta por Quaggio et al. 2005 (Tabela 5).

\section{DISCUSSÃO}

$\mathrm{O}$ desequilíbrio nutricional generalizado de $\mathrm{K}$ em contraste aos demais nutrientes (Tabela 3), justifica-se pelo seu baixo teor encontrado no solo na maioria das glebas monitoradas (Tabela 1). Possivelmente, os baixos teores de K encontrados no solo sejam reflexos da ausência de adubaçôes equilibradas nestas glebas. Este resultado sugere ser o $\mathrm{K} \mathrm{o}$ elemento mais relevante para o manejo da adubaçáo, por ser aquele cujo equilíbrio nutricional estaria sendo mais afetado, principalmente por ser um nutriente com conhecida resposta a sua aplicação em outras regiôes (Quaggio et al. 2011).

A distribuição aleatória para a maioria dos nutrientes (Tabela 3) pode ser explicada pelo fato das normas DRIS serem oriundas do conjunto da populaçáo utilizada para a avaliação nutricional, situação que também já foi constatada para cupuaçueiros cultivados na regiáo amazônica (Wadt $e t$ al. 2011).

De forma geral, observa-se que a FS estimada neste trabalho é invariavelmente menor que a amplitude indicada para os teores dos mesmos nutrientes por outros autores, em especial, para os micronutrientes (Figura 1). Essa pequena amplitude da faixa estimada ocorre devido ao baixo desvio padrão dos teores nutricionais da população nutricionalmente equilibrada (Tabela 4). De forma análoga aos trabalhos de Wadt et al. (1998), Urano et al. (2007) e Serra et al. (2010), a amplitude das FS estimadas pelo método DRIS, foi menor quando comparadas aos valores encontradas na literatura.

As FS estimadas para N, P e Mg encontram-se acima da recomendada pela literatura, com exceçôes das faixas para P proposta por Malavolta et al. (1994) e Mg sugerida por Quaggio et al. (2005) que coincidem com a estimada neste trabalho. Para K, Ca, S, B, Cu, Fe, Mn e Zn as FS determinadas pelo método DRIS foram abaixo do sugerido pela literatura, com exceçáo do $\mathrm{Cu}$ que coincide com a faixa definida por Quaggio et al. (2005) (Tabela 4).

De forma semelhante Camacho et al. (2012) observaram que as $\mathrm{FS}$ estimadas para $\mathrm{K}, \mathrm{Ca}, \mathrm{Mg}, \mathrm{Zn}$ e B pelo método DRIS, chance matemática e pelo NC obtido por meio do critério de distribuiçấo normal reduzida para laranjeiras-pêra foram diferentes das FS recomendada pela literatura.

Diferenças entre padrões nutricionais já foram relatadas na literatura por diferentes autores (Santana et al. 2008; Farnesi et al. 2009) e, se relaciona a diferentes condiçóes

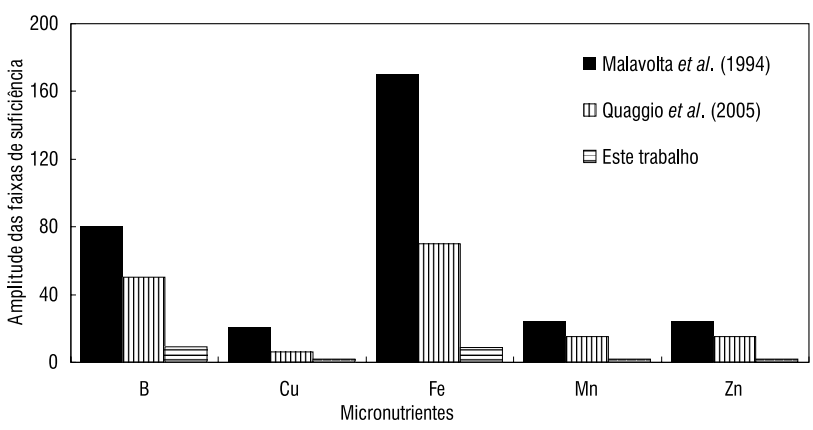

Figura 1 - Amplitude das faixas de suficiência para B, Cu, Fe, Mn e Zn na cultura da laranjeira proposta por dois autores e neste trabalho. 
edafoclimáticas e, ou, manejo da fertilidade do solo. Outro fator pode ser atribuído as diferentes condiçôes experimentais, como observado por Fernandes et al. (2010) e Camacho et al. (2012) na avaliação do estado nutricional de laranjeira-pêra nos estados do Pará e São Paulo, respectivamente.

$\mathrm{O}$ alto teor foliar de $\mathrm{N}$ na maioria das glebas monitoradas pode estar associado à forma empírica como são manejadas estas glebas na regiáo, ou mesmo pelo uso irregular de análise foliar na recomendaçáo de adubação nitrogenada, implicando em uso excessivo de $\mathrm{N}$ nas fertilizaçóes (Tabela 5).

A deficiência generalizada de $\mathrm{P}$ nas glebas possivelmente está atribuído ao baixo teor deste elemento no solo (Tabela 1). Agrava-se este fato a ineficiência da adubaçáo fosfatada, seja pela quantidade insuficiente do nutriente utilizado nas fertilizaçôes ou pela fixação de fósforo, devido as características físico-química dos solos da região (Falcão e Silva 2004).

A baixa capacidade da FS estimada neste trabalho detectar deficiência de K comparativamente as FS da literatura provavalmente está associada as normas DRIS. Pois como os teores foliares de $\mathrm{K}$ foram abaixo de $13 \mathrm{~g} \mathrm{~kg}^{-1}$, a faixa normal estimada tende a ser baixa comparativamente aos padróes nutricionais sugeridos pela literatura, consequentemente diminui a capacidade da FS estimada detectar glebas nutricionalmente deficientes. Isto indica que as adubaçôes aplicadas em todas glebas monitoradas náo fornecem quantidades adequadas de $\mathrm{K}$.

A deficiência generalizada de $\mathrm{K}$ e Ca nestas glebas, possivelmente esta ocorrendo pelo desequilibrio entre $\mathrm{K}, \mathrm{Ca}$ e Mg no solo refletindo em baixos teores de K, Ca e alto teor de $\mathrm{Mg}$ nas folhas. Como estes nutrientes são absorvidos pelos mesmos mecanismos na membrana celular, provavelmente a absorção de $\mathrm{Mg}$ foi preferencial aos demais cátions (Medeiros et al. 2008). Agrava-se ainda para baixos teores de $\mathrm{Ke}$ Ca nas folhas, o fato do $\mathrm{K}$ ser exportado em maior quantidade pelos frutos (Boaretto et al. 2007) e o Ca por ser o elemento de menor mobilidade na planta comparativamente aos demais nutrientes (Duenhas et al. 2007).

A deficiência de $S$ pode estar relacionada a ausência do elemento em fontes de fertilizantes altamente solúveis, em especial o super fosfato triplo, que é comum sua utilização como fonte de P na adubaçáo anual das glebas avaliadas. Ainda quanto ao $S$, é válido inferir que a faixa ótima proposta por Malavolta et al. (1994) foi considerada inadequada para a interpretação do estado nutricional de laranjeiras-pêra na região central de Góias (Santana et al. 2007) e, por ser muito parecida com a FS proposta por Quaggio et al. (2005), ambas FS podem estar diagnosticando uma situação de falsa deficiência.
De forma semelhante a este trabalho, Fernandes et al. (2010) observaram que Cu e Mn também foram limitantes para produção de laranja pêra no estado do Pará. A discordância entre o diagnóstico nutricional proporcionado pelo método DRIS e pelas FS estimadas neste trabalho para $\mathrm{Fe}$ e $\mathrm{Cu}$, justifica-se pelo fato das FS serem oriundas dos teores médios nutricionais da populaçáo nutricionalmente equilibrada. Neste caso, quanto menor o desvio padrão entre os teores nutricionais do conjunto de glebas referência, proporcionalmente será a amplitude da faixa ótima estimada, consequentemente aumenta-se a capacidade da FS na detecção de glebas sob estado de desequilibrio nutricional comparativamante a utilização de FS com maiores amplitudes.

De forma geral a disponibilidade de Fe, $\mathrm{Cu}, \mathrm{Mn}$ e $\mathrm{Zn}$ para laranjeiras é regulada pelas condiçóes do solo, como teor de matéria orgânica, textura e, principalmente o $\mathrm{pH}$, por exemplo quando este último encontra-se em valores mais elevados, ocorre diminuição da solubilização e da absorção destes micronutrientes catiônicos.

Santana et al. (2007) apontam o Zn como o nutriente mais limitante à produção de laranjeiras-pêra na regiáo Goiás, pois sua deficiência seria comum em solos brasileiros, principalmente com a cultivar pêra (Mattos Junior et al. 2001). Quaggio e Piza Junior (2001) também relataram que em condiçôes tropicais, a deficiência de $\mathrm{Zn}$ nos solos é frequente e, têm limitado a produtividade e a qualidade dos frutos cítricos no Brasil.

A deficiência de B tem sido observada frequentemente na citricultura, sendo conseqüência do excesso de chuvas na época da coleta de folhas (fevereiro a abril), reduzindo-se sua disponibilidade para a planta e a alta extração deste nutriente pela cultura, onde nem sempre há reposição mediante adubação (Santana et al. 2007).

No Brasil, B, Zn e Mn são os micronutrientes mais limitantes à produção dos citros, pelos baixos teores no material de origem e pela adsorção específica que ocorre com a matriz de solos cultivados. Períodos prolongados de seca e excesso de chuvas reduzem ainda a absorção de $\mathrm{B}$ pelas plantas (Quaggio et al. 2005).

Embora, na Amazônia central a citricultura encontre-se adaptada ao bioma, a produtividade das glebas ainda é baixa, em torno de $11 \mathrm{t} \mathrm{ha}^{-1}$, se comparado a São Paulo $\left(26 \mathrm{t} \mathrm{ha}^{-1}\right)$, maior produtor nacional (IBGE 2011). Um dos fatores pode estar relacionado ao manejo inadequado das fertilizaçóes, sendo agravado pela ausência ou insuficiências de práticas conservacionistas para o manejo do solo. Neste sentido, a disponibilização de NC e FS obtidas de laranjeiras cultivada nestas condições contribui para melhorar o monitoramento das adubaçóes e, ao manejo da fertilidade do solo nestas glebas. 


\section{CONCLUSÕES}

Para maioria dos nutrientes, os valores nutricionais estimados não foram concordantes com os valores da literatura e grande parte das glebas monitoradas encontram-se em desequilibrio nutricional, sendo que em quase $50 \%$ destas, $\mathrm{P}$, $\mathrm{K}, \mathrm{Ca}, \mathrm{S}, \mathrm{B}, \mathrm{Cu}$ e Fe estấo abaixo dos níveis críticos propostos neste trabalho. Isto sugere que os produtores de laranja na Amazônia Central deveriam atentar-se para estes elementos no planejamento das fertilizaçóes.

Face ao elevado custo em tempo, recursos humanos e técnicos para o desenvolvimento de ensaios de calibração para obtenção tradicional de valores referência, o método DRIS mostra-se como alternativa promissora para determinação de NC e FS para laranjeiras, principalmente na ausência de informaçōes nutricionais.

\section{AGRADECIMENTOS}

Ao Projeto PI-Citros/FAPEAM pelo auxílio à pesquisa.

\section{BIBLIOGRAFIA CITADA:}

Beverly, R.B. 1993. DRIS Diagnoses of soybean nitrogean, phosphorus, and potassium status are usatisfactory. Journal of Plant Nutrition, 16:1431-1447.

Boaretto, R.M.; Mattos Junior, D.; Trivelin, P.C.O.; Muraoka, T.; Boaretto, A. E. 2007. Acúmulo de nutrientes e destino do nitrogênio $\left({ }^{15} \mathrm{~N}\right)$ aplicado em pomar jovem de laranjeira. Revista Brasileira de Fruticultura, 29: 600-605.

Camacho, M. A.; Silveira, M. V. da; Camargo, R. A.; Natale, W. 2012. Faixas normais de nutrientes pelos métodos ChM, DRIS e CND e nível crítico pelo método de distribuição normal reduzida para laranjeira-pera. Revista Brasileira de Ciência do Solo, 36: 193-200.

Duenhas, H.L.; Villas Boas, R.L. Souza, C.M.P.; Oliveira, M.V.A.M.; Dalri, A.B. 2005. Produçáo, qualidade dos frutos e estado nutricional de laranja valência sob fertirrigação e adubação convencional. Engenharia Agricola, 25: 154-160.

Fageria, N.K.; Barbosa Filho, M.P.; Moreira, A.; Guimarães, C.M. 2009. Foliar fertilization of crop plants. Journal of Plant Nutrition, 32: 1044-1064.

Falcão, N.P. de S.; Silva, J.R.A. 2004. Características de adsorção de fósforo em alguns solos da Amazônia Central. Acta Amazônica, 34: 337-342.

Farnesi, M. de M.; Silva, E. de B.; Guimarães, P.T.G. 2009. Diagnose nutricional de cafeeiros da região do alto Jequitinhonha (MG): normas DRIS e faixas críticas de nutrientes. Revista Brasileira de Ciência do Solo, 33: 969-978.

Fernandes, A.R.; Reis, I.N.R.S.; Noronha, N.C. 2010. Estado nutricional de pomares de laranjeira submetidos a diferentes manejos do solo. Revista de Ciências Agrárias, 53: 52-58.
Instituto Brasileiro de Geografia e Estatística - IBGE. 2011. Produçâo agrícola municipal 2010: culturas temporárias e permanentes. IBGE, Rio de Janeiro. 91pp.(http://www.ibge.gov. $\mathrm{br} /$ estadosat/temas.php?sigla=am\&tema=lavourapermanten2010). Acesso em 04/03/2012.

Kurihara, C.H.; Maeda, S.; Alvarez V., V.H. 2005. Interpretação de resultados de análise foliar. Embrapa Agropecuária Oeste; Colombo; Embrapa Florestas, Dourados, Mato Grosso do Sul. 42pp. (Documentos, 74).

Lana, R.M.Q.; Oliveira, S.A.; Lana, A.M.Q.; Faria, M.V. 2010. Levantamento do estado nutricional de plantas de Coffea arabica L. pelo DRIS, na regiấo do Alto Paranaíba - Minas Gerais. Revista Brasileira de Ciência do Solo, 34: 1147-1156.

Malavolta, E. Prates, H.S.; Casale, H.; Leão, H.C. 1994. Seja o doutor dos seus citros. Potafós, Piracicaba, São Paulo. 22pp. (Informações Agronômicas, 65).

Malavolta, E.; Vitti, G.C.; Oliveira, S.A. de. 1997. Avaliação do estado nutricional de plantas: princípios e aplicaçôes. 2 ed., rev. e atual. Potafós, Piracicaba, Sáo Paulo, 319pp.

Mattos Junior, D.; Quaggio, J.A.; Cantarella, H. 2001. Calagem e adubação dos citros. Informe Agropecuário, 22: 39-46.

Medeiros, J.C.; Albuquerque, J.A.; Mafra, A.L.; Rosa, J.D.; Gatiboni, L.C. 2008. Relação cálcio:magnésio do corretivo da acidez do solo na nutrição e no desenvolvimento inicial de plantas de milho em um Cambissolo Húmico Álico. Semina: Ciências Agrárias, 29: 799-806.

Parent, L.E. 2011. Diagnosis of the nutrient compositional space of fruit crops. Revista Brasileira de Fruticultura, 33: 321-334.

Quaggio, J.A.; Mattos Junior, D.; Boaretto, R. M. 2011. Sources and rates of potassium for sweet orange production. Scientia Agricola, 68: 369-375.

Quaggio, J.A.; Mattos Junior, D.; Cantarella, H. 2005. Manejo da fertilidade do solo na citricultura, p. 483-507. In: Mattos Junior, D.; De Negri, J.D.; Pio, R.M.; Pompeu Junior, J. (Eds). Citrus. Instituto Agronômico de Campinas, Campinas, São Paulo.

Quaggio, J.A.; Piza Junior, C.T. 2001. Micronutrientes para frutíferas tropicais, p. 459-491. In: Ferreira, M.E.; Cruz, M.C.P.; Raij, B. Van; Abreu, C.A. (Eds). Micronutrientes tóxicos e metais pesados na agricultura. Potafós/Fapesp/CNPq, Jaboticabal, São Paulo.

Santana, J. das G.; Leandro, W.M.; Naves, R.V.; Cunha, P.P. 2008. Normas DRIS para interpretação de análises de folha e solo, em laranjeira pêra, na regiāo central de Goiás. Pesquisa Agropecuária Tropical, 38: 109-117.

Santana, J. das G.; Leandro, W.M.; Naves, R.V.; Cunha, P.P.; Rocha, A.C. 2007. Estado nutricional da laranja pêra na região central do estado de Goiás avaliada pelas análises foliar e do solo. Bioscience Journal, 23: 40-49.

Serra, A.P.; Marchetti, M.E.; Vitorino, A.C.T.; Novelino, J.A.; Camacho, M.A. 2010. Determinação de faixas normais de nutrientes no algodoeiro pelos métodos ChM, CND e DRIS. Revista Brasileira de Ciência do Solo, 34: 97-194. 
Sistema de Proteção da Amazônia - SIPAM. 2005. Boletim climático da Amazônia. Divisão de metereologia, Manaus, Amazonas. 2pp.

Urano, E.O.M.; Kurihara, C.H.; Maeda, S.; Vitorino, A.C.T.; Gonçalves, M.C.; Marchetti, M.E. 2007. Determinação de teores ótimos de nutrientes em soja pelos métodos chance matemática, sistema integrado de diagnose e recomendação e diagnose da composição nutricional. Revista Brasileira de Ciência do Solo, 31: 63-72.

Wadt, P.G.S. 2005. Relationships between soil class and nutritional status of coffee plantations. Revista Brasileira de Ciência do Solo, 29: 227-234.
Wadt, P.G.S.; Dias, J.R.M.; Perez, D.V.; Lemos, C.de O. 2011. Fórmulas DRIS para o diagnóstico nutricional de pomares de cupuaçueiros. Bragantia, 70: 649-656.

Wadt, P.G.S.; Novais, R.F.; Alvarez V., V. H.; Fonseca, V.; Barros, N.F. 1998. Valores de referência para macronutrientes em eucalipto obtidos pelos métodos DRIS e chance matemática. Revista Brasileira de Ciência do Solo, 22: 685-692.

Recebido em: 09/02/2012

Aceita em: 11/07/2012 